\title{
Diagnosis and Treatment of Halitosis: An Overview
}

Vineet Vaman Kini, Richard Pereira, Ashvini Padhye, Sachin Kanagotagi, Tushar Pathak, Himani Gupta

\begin{abstract}
'Halitosis' or bad breath is an unpleasant problem that affects people socially and psychologically. Halitosis is caused by a mixture of breath with malodorous compounds emanating from different areas of the oral cavity, respiratory tract and upper digestive tracts.

Breath odor research captured the scientific community's attention during the last few decades. This has led to advances in analytical instruments used for identification and measurement of these malodorous compounds. The dental profession's response to the problem of halitosis has been met with hurdles in regards therapy often due to perceptive differences of the patient. This review attempts to highlight the identification, classification, diagnosis and treatment of halitosis.
\end{abstract}

Keywords: Halitosis, Organoleptic scoring, Volatile sulfur compounds, Sulfide monitoring, Instrumentation.

How to cite this article: Kini VV, Pereira R, Padhye A, Kanagotagi S, Pathak T, Gupta H. Diagnosis and Treatment of Halitosis: An Overview. J Contemp Dent 2012;2(3):89-95.

Source of support: Nil

Conflict of interest: None declared

\section{IDENTIFICATION OF HALITOSIS}

Identification of halitosis or 'bad breath' is in itself a problem due to subjectivity of perception of the examiner and the patient. ${ }^{1}$ People often are unaware of their own bad breath. ${ }^{1,2}$ Our inability to smell our own oral malodor has been attributed to adaptation or dulling of sensation resulting from continual exposure. This lack of objectivity poses a hurdle not only during diagnosis but also on end results of therapy especially when a psychological angle of etiopathogenesis is identified, i.e. pseudohalitosis and halitophobia. ${ }^{3,4}$ Pseudohalitosis is where the oral malodor does not exist, but the patient believes that he/she has it. If after successful treatment for either genuine halitosis or pseudohalitosis the patient still believes that he/she has halitosis then the diagnosis is termed halitophobia. ${ }^{3,4}$

\section{Organoleptic/Hedonic Method}

The human nose is best method to perceive malodor. This method identifies the malodorous compounds qualitatively and to an extent semiquantitatively through scoring (Table 1). ${ }^{4}$ This requires that patients do not consume any odorous foods and abstain from smoking and alcohol prior to examination by an odor judge subjected to the same precautions. It provides us with the identification of 'true halitosis' caused by pathology be it by an intraoral or extraoral source. Caution should be maintained for airborne infections.

\section{Questionnaire}

This serves as a tool for assessing the social interactions of the patient and is expected to interrogate the subject on whether the halitosis was self-perceived or brought to attention by family and friends. This would highlight social problems created for the patient by halitosis and in addition would bring out confounding factors to diagnosis, such as pungent diets, alcohol and tobacco consumption. This would be critical in establishing the grounds for pseudohalitosis and halitophobia. ${ }^{5}$

\section{ETIOPATHOGENESIS OF HALITOSIS}

The malodorous compounds leading to halitosis are indicative of underlying diseases and metabolic disorders that need to be identified and taken into consideration. ${ }^{6-9}$ These conditions lead to true or genuine halitosis which is attributed to pathology warranting an interdisciplinary approach for successful therapy. Halitosis is due to the presence of odorous gases in the air expelled from the oral cavity. These compound were found to be gaseous in nature, i.e. volatile sulfur compounds (VSCs) namely hydrogen sulfide, methyl mercaptan, dimethyl sulfide, dimethly disulfide, allyl mercaptan, allyl methyl sulfide, propyl mercaptan, methyl propyl sulfide, carbon disulfide, ammonia, dimethylamine and trimethylamine to name a few. ${ }^{10-12}$ VSCs are mainly produced through putrefactive activities of bacteria present in saliva, the gingival crevice, the tongue surface and other areas (Flow Chart 1). The substrates are sulfur-containing amino acids, such as cysteine, cystine and methionine, which are found free in saliva, gingival crevicular fluid or produced as a result of proteolysis of protein substrates. The nature of these compounds lead to the quality of the malodor (Table 2). ${ }^{10-12}$ The putrefactive changes can occur in both physiology and pathology leading to physiological halitosis and pathological halitosis respectively. Clinical examination is needed to determine whether the pathology is of intraoral or extraoral origin.

\section{CLASSIFICATION OF HALITOSIS}

Classification is not only imperative to understand etiopathogenesis but also for therapy especially when interdisciplinary referral is warranted. 
Table 1: Organoleptic scoring of halitosis

\section{Category}

0 : Absence of odor

1: Questionable odor

2: Slight malodor

3: Moderate malodor

4: Strong malodor

5: Severe malodor

\section{Description}

Odor cannot be detected

Odor is detectable, although the examiner could not recognize it as malodor

Odor is deemed to exceed the threshold of malodor recognition

Malodor is definitely detected

Strong malodor is detected, but can be tolerated by examiner

Overwhelming malodor is detected and cannot be tolerated by examiner (examiner instinctively averts the nose)

\begin{tabular}{|ll|}
\hline \multicolumn{2}{|c|}{ Table 2: VSCs odor } \\
\hline Name & Odor qualification \\
\hline Hydrogen sulfide & Rotten eggs \\
Methyl mercaptan & Pungent, rotten cabbage \\
Dimethyl sulfide & Unpleasantly sweet \\
Allyl mercaptan & Garlic like \\
Allyl methyl sulfide & Garlic like \\
Carbon disulfide & Slightly pungent \\
Ammonia & Pleasantly sweet \\
Dimethylamine & Fishy, ammonical \\
Trimethylamine & Fishy, ammonical \\
\hline
\end{tabular}

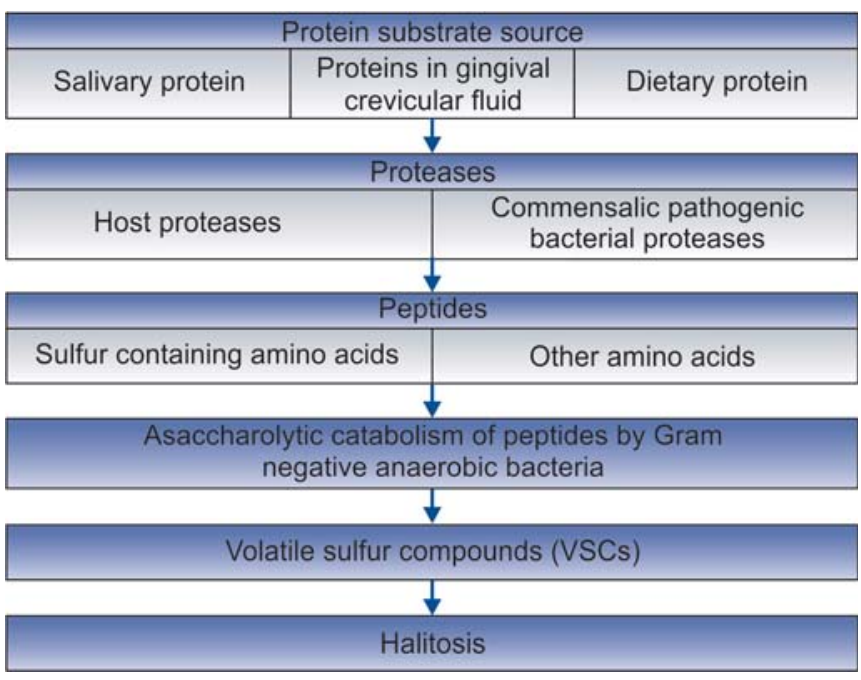

Flow Chart 1: Production of VSCs by proteolytic bacteria causing malodor

In 1954, Attia et al classified halitosis comprising all factors contributing to the etiology of halitosis and encompassing multiple disciplines. ${ }^{6,13,14}$ It highlighted respiratory, gastrointestinal and systemic diseases also indicating the neurological and psychological aspects of breath malodor. Lu Dominic in 1982 classified true halitosis into physiological halitosis and pathological halitosis. ${ }^{13,14}$ The major drawback in this classification was that etiologies seemed to overlap in some instances particularly in reference to halitosis due to systemic causes.

Yaegaki K and Coil JM in 2000 presented a classification of halitosis (Table 3) based on the corresponding treatment needs for that existing condition with corresponding treatment needs encompassing all etiologies of halitosis including physiologic, pathologic (both intraoral and extraoral causes) and highlighted the psychological parameters of halitosis as in pseudohalitosis and halitophobia. ${ }^{13,14}$

\section{EXAMINATION OF THE PATIENT FOR HALITOSIS}

\section{Intraoral Examination}

It is carried out for intraoral pathologic halitosis and niches for putrefactive econiches that contribute toward production of VSCs. ${ }^{7,9,15,16}$ Examination includes examination of the teeth for unrestored caries, endodontic infections, overhanging restorations and ill-fitting prosthesis which can lead to plaque accumulation, periodontal disease and food lodgment that provide substrate to the intraoral pathogens. The intraoral soft tissue econiches include the dorsum of the tongue, gingival sulci/periodontal pockets, the tonsils and floor of the mouth. ${ }^{17-20}$

\section{THE DORSUM OF THE TONGUE}

The examination of the dorsum of the tongue should include:

- Examination for deep tongue fissures and prominent sulcus terminalis for tongue coats.

- Presence and location of the tongue coats and the thickness and color of same indicating duration and dietary influence on the same respectively.

- The presence or absence of postnasal drip residues precipitating on the posterior part of the dorsum of the tongue.

- Vigil for ulcers, erosions and pinpoint bleeding areas indicating duration and desquamative disease, Candida, etc.

- Presence of tonsiloliths migrating to the posterior part of the dorsum of the tongue.

Scraping the tongue with a spoon and smelling the scraping can attribute the role of the tongue coat in contributing toward halitosis. Tongue coat indices and tongue coat weight analysis can act as adjunctive tools. ${ }^{20,21,23,24}$

Chairside tests, such as Halitox ${ }^{\mathrm{TM}}$ are useful as semiquantitative tests for both VSCs, such as hydrogen sulfide and methyl mercaptan, as well as polyamines like 


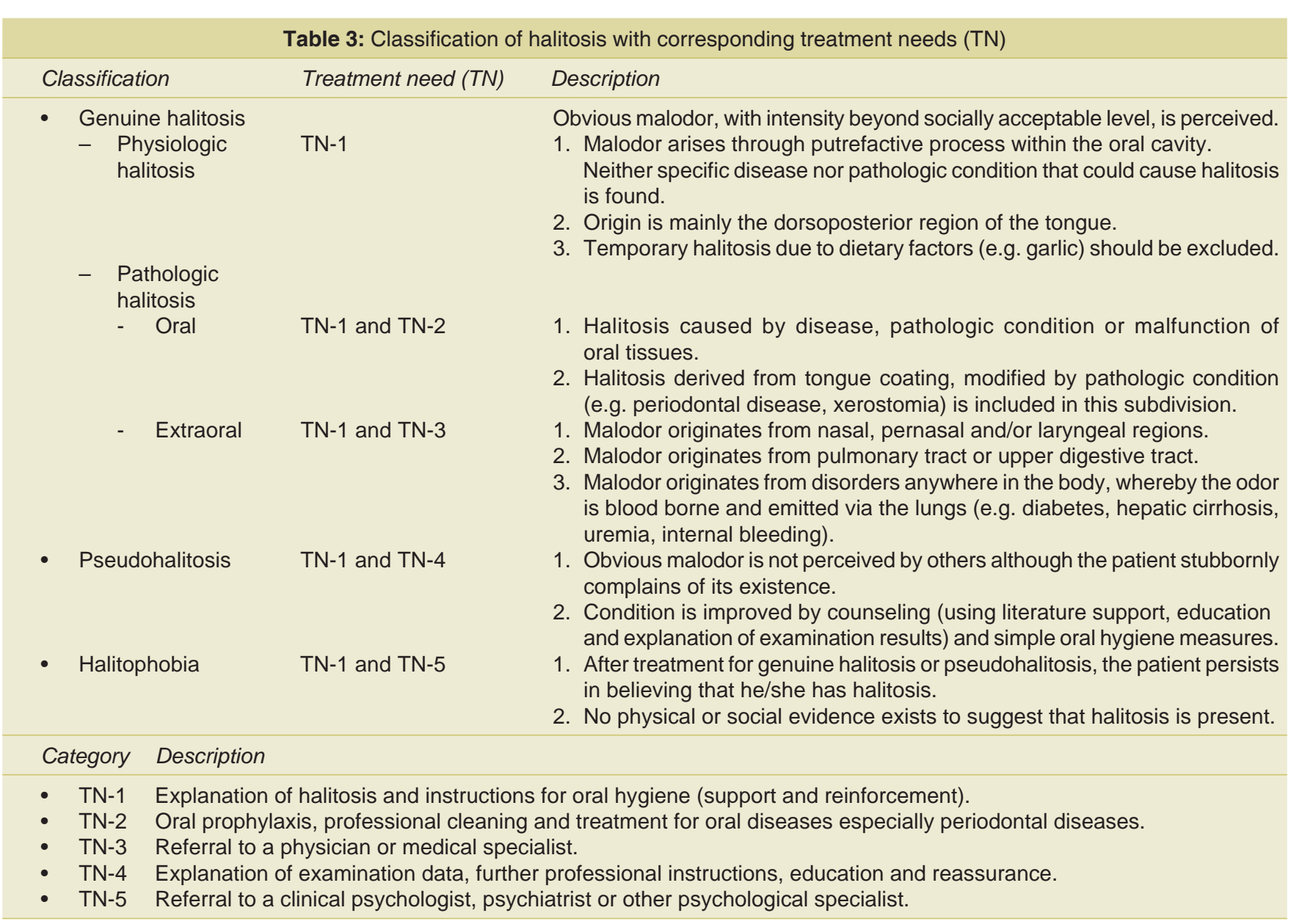

putrescine and cadaverine based on its color changes when exposed to a sample of tongue scraping. The intensity of hue change from colorless to yellow to yellowish brown depicts loosely both the number of VSC-producing bacteria and the intensity of VSC produced which can be correlated with the quantity of tongue coat clinically present to draw clinical inferences. ${ }^{22-24}$

\section{GINGIVAL SULCUS/PERIODONTAL POCKET EXAMINATION}

Evidence shows that apart from the dorsum of the tongue, the gingival sulcus/pseudopocket and the periodontal pocket is the next econiche which is capable of harboring VSCproducing proteolytic anaerobic bacteria. In fact it was proposed that the periodontal pathogens through transechonical migration used to colonize the dorsum of the tongue contributing equivocally to halitosis.

The proteolytic periodontal pathogens involved in this process are Treponema denticola, Porphyromonas gingivalis and Tannerella forsythia which give a positive enzymatic benzoyl-DL-arginine-2-naphthylamide (BANA) test due to their trypsin like proteases, gingipains. ${ }^{24,25}$

The VSCs produced by these periodontal pathogens within the periodontal pocket/gingival sulcus can be measured by a specially designed periodontal probe, the Perio 2000 Diamond probe system.

\section{OTHER INTRAORAL ECONICHES}

Other intraoral econiches that can be involved in putrefactive proteolytic processes include the lining mucosa, floor of the mouth and hard palate mucosa and their putrefactive products are accumulated in the saliva. The saliva collected from the mouth can be collected and tested for tissue breakdown products by TOPAS ${ }^{\mathrm{TM}}$ — toxicity prescreening assay for hydrogen sulfide and methyl mercaptan along with polyamines, such as putrescine and cadaverine, cystiene tolerance test for cysteine breakdown products in saliva using the salivary supernatant sediment test (SSS system) and The Swinnex filter test to determine the malodorous potential of stagnated saliva as in the stagnant saliva during nocturnal conditions contributing toward morning breath. ${ }^{22-25}$

\section{EXTRAORAL EXAMINATION}

Interdisciplinary referrals to physicians are warranted in the case of extraoral pathologic halitosis. The examinations would include complete systemic examination and examination of organ systems specific to the problems of 
malodor. ${ }^{26}$ These may include certain procedures, such as laryngoscopy, gastrointestinal endoscopy, routine radiographic procedure and laboratory diagnostic procedures to detecting systemic condition, which may have an influence on halitosis, i.e. blood borne halitosis. Their opinion on the various systemic conditions influence on halitosis is indispensable while treating the patient as a whole and should be given priority, if warranted to direct the patient to a more medically oriented treatment plan rather than just a dentally oriented treatment plan. Moreover, their evaluation may lead to more insight on psychiatric profile of the patient and may make the road to referral to a psychiatric specialist in cases of pseudohalitosis and halitophobia. ${ }^{27}$

Extraoral causes of halitosis include:

Respiratory tract lesions like sinusitis, foreign bodies, atrophic rhinitis (ozena), Wegener's granulomatosis, tuberculosis, rhinoscleroma, adenoiditis, nasopharyngeal abscess, carcinoma of the larynx, laryngoscleroma, pulmonary abscess, carcinoma of the lung, bronchiectasis, necrotizing pneumonitis and empyema may all be associated with halitosis. ${ }^{6,26,27}$

Gastrointestinal conditions like salivary gland dysfunction, retropharyngeal abscess, Zenker's diverticulum, congenital bronchoesophageal fistula, gastric carcinoma, hiatus hernia and pyloric stenosis, as well as with enteric infections can cause halitosis. However, the current view is that halitosis, if present in conjunction with these disorders, is actually caused by disorders of the oral cavity. The unpleasant odor emitted from the lower gastrointestinal tract is only detectable during retching or vomitting, because the esophagus is normally collapsed., ${ }^{6,26,27}$

Patients with neurological conditions that cause a disordered sense of smell (dysosmia) may believe that they have halitosis; this is referred to as subjective halitosis because other people cannot detect an odor. ${ }^{6,26,27}$

Subjective halitosis, i.e. halitophobia and pseudohalitosis may occur as a manifestation of a mental disorder, usually a psychosis..$^{27,28}$ For example, if patients complain of 'rotten breath' and say that their stomach and lungs are rotting away they most probably more often than not have a form of psychosis. Such patients may also complain of foul odors around them. An examination of mental status will confirm the diagnosis. In these cases, treatment with major tranquilizers is usually necessary. In patients with depression, halitosis is usually just one of the many somatic complaints. $^{27,28}$

\section{MEASUREMENT OF HALITOSIS}

1. Organoleptic measurement: As described before it is a subjective test scored on the basis of the examiner's perception of a subject's oral malodor. However it is not quantitative. ${ }^{29}$

2. Gas chromatography: Gas chromatography (GC) is considered the gold standard for measuring oral malodor since it is specific for VSCs, the main cause of oral malodor. The GC equipment is expensive, bulky and the procedure requires a skillful operator. Therefore, this technology has been confined to research and not to clinical use. ${ }^{3,30}$

3. Sulfide monitoring: Sulfide monitors analyze for total sulfur content of the subject's mouth air. Although compact sulfide monitors are inexpensive, portable and easy to use, most of them are not able to distinguish among the VSCs. For example, the Halimeter ${ }^{\mathrm{TM}}$ (Interscan Co., Chatsworth, CA) has high sensitivity for hydrogen sulfide but low sensitivity for methyl mercaptan, which is a significant contributor to halitosis caused by periodontal disease. ${ }^{31,32}$

4. Ammonia detector: For measuring malodorous substances not having sulfide like (indole, skatole, putrescine and cadaverine which are ammoniferous compounds and cause halitosis). ${ }^{31-33}$

The most reliable and practical procedure for evaluating a patient's level of oral malodor is still done through an organoleptic assessment by a trained clinician. Nevertheless, the use of a portable sulfide monitor is of interest, since we can quantify the changes and the patients are able to monitor their evolution through therapy. This is an important factor especially in those patients with pseudohalitosis or halitophobia. ${ }^{27,28}$

\section{TREATMENT OF HALITOSIS}

\section{Physiologic Halitosis}

Practical treatment of physiologic halitosis requires TN-1. Since tongue coating is comprised of desquamated epithelial cells, blood cells and bacteria; cleaning the tongue reduces VSCs. Although brushing and flossing are not very effective in reducing oral malodor, these procedures are required to maintain good oral hygiene, and to prevent periodontal conditions, which are the most frequent causes of oral pathologic halitosis. Some kinds of mouthwashes and toothpastes are also effective in reducing oral malodor (Table 4) ${ }^{34-37}$ However, practitioners must refer to articles published in peer reviewed journals to determine whether a product is effective in reducing oral malodor. When evaluating the literature it should be noted that in vitro study of products is impractical for examining their ability to reduce oral malodor; the effects must be investigated in vivo as well. Organoleptic measurement of oral malodor is frequently employed to determine the clinical efficacy of 
Table 4: Effectiveness of rinses used for the reduction of oral malodor

Method

Rinsing with water

Use of sanguinarine rinses

Essential phenolic oils

Zinc chloride rinses

Two-phase mouthwash

Chlorhexidine

Chloride dioxide

Cetylpyridinium chloride
Effectiveness in reduction of oral malodor

Effective for 15 minutes.

No detectable decreases have been reported.

Low substantivity and only transient antibacterial effects, but measurable reduction.

Marked reduction of VSC levels overtime. Ionic zinc inhibits VSCs for 10 hours, reduces odor by $71 \%$.

Oil, water and cetylpyridinium chloride, found very effective at full strength.

Substantive antimicrobial agent, effective against both Gram negative and Gram positive bacterial species some unfortunate side effects, such as staining and bitter taste.

No research to show efficacy or long-term effects. Some caution from a report by the Canadian cancer research with respect to chlorine dioxide in the water supply.

Shown to reduce VSC production for 3 hours. the products, but the measurement is neither objective nor scientific. $^{34}$

Reliable clinical research articles are available only on mouthwashes containing zinc, chlorhexidine, highly concentrated alcohol and hydrogen peroxide, demonstrating their efficacy in reducing malodor. Dental prophylaxis is one of the most important of TN-1 measure, not only halitosis patients but also dental patients must be instructed to have regular examination and scaling. ${ }^{34,35}$

\section{ORAL PATHOLOGIC HALITOSIS}

As the presence of periodontal conditions is a main contributor to oral pathologic halitosis, periodontal treatment is frequently required. Hence, regular dental treatments such as restorations, root-canal treatment or extractions are required for these cases. A chronic ulcer in the oral cavity, such as a cancer, is a very rare cause of halitosis. Since a large reduction of salivary secretion causes oral pathologic halitosis, the treatment of xerostomia may reduce oral malodor. It was found that residual saliva on the posterior palate is specifically reduced in hyposalivators. So-called 'jungle breath' or 'jungle mouth' is considered to be caused by reduced salivation during sleeping. Specific treatment of this etiology with sialagogues has also been indicated for the rectification of oral malodor. $6,34,35$

\section{EXTRAORAL PATHOLOGIC HALITOSIS}

Extraoral pathologic halitosis treatments are outside the realm of dental practitioners. If practitioners diagnose or suspect this condition, they should quickly refer a patient to a medical practitioner (TN-3). ${ }^{6,27,28}$

\section{MANAGEMENT OF PSEUDOHALITOSIS AND HALITOPHOBIC PATIENTS}

Pseudohalitosis and halitophobia patients believe they have halitosis, even though offensive oral malodor is absent. A typical symptom of these conditions is that they interpret other people's behavior, such as 'covering the nose', 'averting the face' or 'stepping back' as an indication that they have oral malodor. Pseudohalitosis patients can accept the practitioner's diagnosis that oral malodor does not exist after having undergone treatment and being reinforced scientific literature support, education and explanation of examination results (TN-4). ${ }^{6,27,28}$

The principle of the management protocol is as follows:

- The practitioner must display attitudes of acceptance, sympathy, support and assurance toward the patient to establish rapport between him/her and the patient.

- The practitioner should not argue with the patient as to whether their oral malodor exists or not.

- The practitioner must explain that other individual's avoidance behavior is not caused by oral malodor.

- The patient must be instructed in TN-1.

- The patient must be instructed that he/she must avoid judging his/her oral malodor by other people’s attitude. Halitophobic patients are quite unhappy with their dental practitioners, who diagnose no oral malodor. Sometimes practitioners may lose their rapport with these patients. If practitioner tries to convince a patient to visit a psychological specialist (TN-5) on account of halitophobia, many patients will refuse the referral because they have no doubt that they have severe oral malodor. They still judge their oral malodor by other individuals' attitudes. Therefore, we counsel that a patient needs psychological assistance to avoid judging his/her breath by other people's gestures rather than the issue of malodor itself. If they cannot accept the referral to a specialist, some patients might develop a personality disorder, which is totally outside the realm of dental treatment. ${ }^{6,27,28}$

\section{SUMMARY AND CONCLUSION}

With the ingress of technology and molecular sciences it was proved beyond doubt that oral malodor had its roots both in the oral cavity as well as other communicating spaces (GIT, upper and lower respiratory tracts and through blood circulation). This warranted a new classification of oral malodor comprising of both intraoral as well as extraoral 


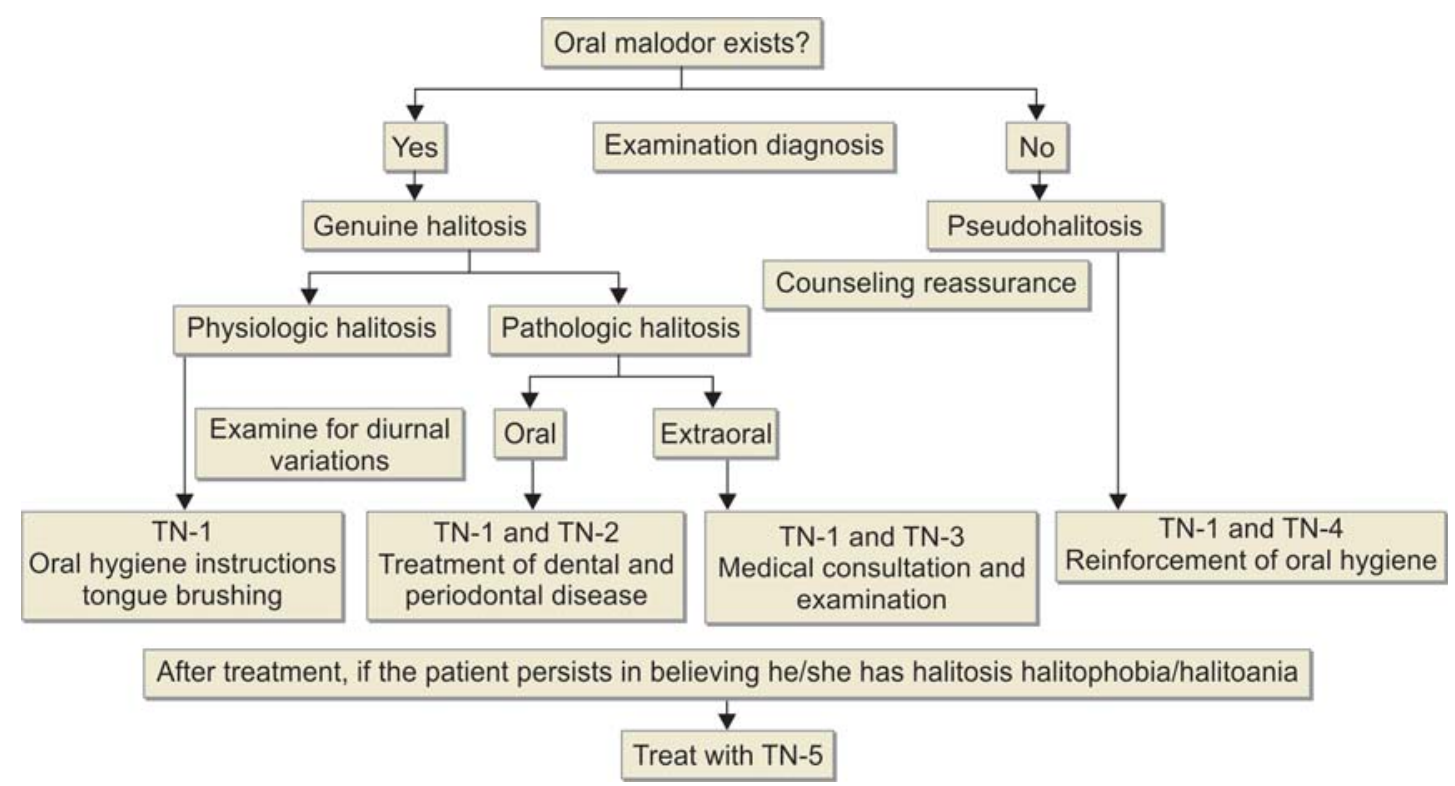

Flow Chart 2: Treatment protocol for halitosis

etiologies and emphasis on treatment needs based on contingency management of the disease (Ken Yaegaki, JM Coil et al) which was not reflected on previously.

In addition to organoleptic assessment of the oral malodor, the invention of sophisticated instruments, i.e. gas chromatographs, portable sulfide monitors and ammonia detectors; science has enabled us to realize the measurement of oral malodor as a reality.

The entry of chairside enzymatic tests and assays have been a very encouraging boost in the direction of recognizing oral malodor with a causative putative pathogen.

Although the current treatment modalities have rendered the clinician to cope with the problem of oral malodor, the success of therapy is subject to patient perception. Therefore, the psychological aspect of the disease should be considered to ensure effective therapy (Flow Chart 2).

\section{REFERENCES}

1. Boxy A. Oral malodor: Philosophical and practical aspects. J Can Dent Assoc 1997;63:196-201.

2. Ibua E, Bhat R. Self-perception of breath odor. J Am Dent Assoc 2001;132:621-26.

3. Rosenberg M. Clinical assessment of bad breath: Current concepts. J Am Dent Assoc 1996;127:475-82.

4. Rosenberg M, McCullochi C. Measurement of oral malodor: Current methods and future prospects. J Periodontol 1992;63: 776-82.

5. Yaegaki K. Clinical application of a questionnaire for diagnosis and treatment of halitosis. Quintessence Int 1999;30:302-06.

6. Attia EL, Marshall KG. Halitosis. Can Med Assoc J 1982;126: 128-35.

7. De Boever EH, Loesche WS. Assessing contribution of anerobic microflora of the tongue to oral malodor. J Am Dent Assoc 1995;126:1384-93.

8. Kleinberg DM, Westbay G. Salivary and metabolic factors involved in oral malodor formation. J Periodontol 1992;63: 768-75.
9. McDowell JD, Kassebamn DK. Diagnosis and treating halitosis. J Am Dent Assoc 1993;124:55-64.

10. Goldberg S, Kozlavsky A, Gordon D. Cadaverine as a putative component of oral malodor. J Dent Res 1994:73:1168-72.

11. Kozlovsky D, Gordon. Correlation between the BANA test and oral malodor parameters. J Dent Res 1994;73:1036-42.

12. Gonerman A. Halitosis in medicine: A review. Int Dent J 2002;52:201-06.

13. Yaegaki K, Coil JM. Examination, classification and treatment of halitosis, clinical perspective. J Can Dent Assoc 2000;66: 257-61.

14. Murata T, Yamage T. Classification and examination of halitosis. Int Dent J 2002;52:181-86.

15. Yaegaki K, Coil JM. Tongue brushing and mouth rinsing as basic treatment measures for halitosis. Int Dent J 2002;52: 192-96.

16. Quiry M, Nan C, Maongardini. The effect of a 1-stage full mouth disinfection on oral malodor and microbial colonization of the tongue in periodontitis patients: A pilot study. J Periodontol 1998;69:374-82.

17. De Boever EH, De Vzeda M. Relationship between volatile sulphur compounds, BANA hydrolyzing bacteria and gingival health in patients with and without complaints of oral malodor. J Am Dent Assoc 1995;126:1393-97.

18. Morita M, Wang HL. Relationship between sulcular sulphide level and oral malodor in subjects with periodontal disease. J Periodontol 2001;72:79-84.

19. Morita M, Wang HL. Relationship of sulcular sulphide level to severity of periodontal disease and BANA test. J Periodontol 2001;72:74-78

20. Nakano Y, Yoshimura M. Methyl mercaptan production by periodontal bacteria. Int Dent J 2002;52:217-20.

21. Morita M, Wang HL. Association between oral malodor and adult periodontitis: A review. J Clin Periodontol 2001;28:813-19.

22. Awano S, Gohara K, Kurihara E. The relationship between the presence of periodontopathogenic bacteria in saliva and halitosis. Int Dent J 2002;52:212-16.

23. Vaneda M, Countreasas A, Chen C. The utility of whole saliva to detectable oral presence of periodontopathic bacteria. J Periodontol 1998;69:828-33. 
24. Louis ZG. Oral malodor: A scientific perspective. J Can Dent Assoc 1993;59:607-10.

25. Ratcliff PA, Johnson PW. The relationship between oral malodor, gingivitis and periodontitis: A review. J Periodontol 1997;70:485-89.

26. Kleinberg DM, Codipilly. Cysteine challenge testing: A powerful tool for examining oral malodor processes and treatments in vivo. Int Dent J 2002;52:221-28.

27. Preti G, Clark L, Cowart BJ. Nonoral etiologies of oral malodor and altered chemosensation. J Periodontol 1992;63:791-96.

28. Yaegaki K, Coil JM. Clinical dilemmas posed by patients with psychosomatic halitosis. Quintessence Int 1999;30:328-33.

29. Rosenberg M, Gelernter I, Barki M. Measurement of halitosis: Current methods and future prospects. J Periodontol 1992;63: 776-82.

30. Furue J, Majms G, Lenton. Comparison of volatile sulphur compound concentrations measured with a sulphide detector vs gas chromatography. J Dent Res 2002;819:140-43.

31. Oho T, Yashida Y. Characteristics of patients complaining of halitosis and gas chromatography for diagnosing halitosis. Oral Surg Oral Med Oral Pathol Oral Radiol Endo 2001;91: 531-34.

32. Rosenberg M, Kulkarni B. A reproducibility and sensitivity of oral malodor measurement, with portable sulphide monitor. J Dent Res 1991;11:1436-40.

33. Rosenberg M, Septon I, Eli I, et al. Halitosis measurement by an industrial sulphide monitor. J Periodontol 1991;62:487-89.

34. Coil JM and Yaegaki K. Treatment needs (TN) and practical remedies for halitosis. Int Dent J 2002;52:187-91.

35. Rolla G, Ski GJ. The significance of the source of zinc and its anti VSC effect. Int Dent J 2002;52:233-35.

36. Rosenberg M, Geleruter I, Barki M. Day long reduction of oral malodor by a two phase oil: Water mouthrinse, as compared to chlorhexidine and placebo rinses. J Periodontol 1992;63:39-43.
37. Loesche WJ. The effects of antimicrobial mouthrinses on oral malodor and their status relative to US Food and Drug Administration regulations. Quintessence Int 1999;30:311-18.

\section{ABOUT THE AUTHORS}

\section{Vineet Vaman Kini (Corresponding Author)}

Reader, Department of Periodontics, MGM Dental College and Hospital Navi Mumbai, Maharashtra, India, e-mail: drvinkin@gmail.com

\section{Richard Pereira}

Professor, Department of Periodontics, MGM Dental College and Hospital, Navi Mumbai, Maharashtra, India

\section{Ashvini Padhye}

Professor and Head, Department of Periodontics, MGM Dental College and Hospital, Navi Mumbai, Maharashtra, India

\section{Sachin Kanagotagi}

Senior Lecturer, Department of Periodontics, MGM Dental College and Hospital, Navi Mumbai, Maharashtra, India

\section{Tushar Pathak}

Senior Lecturer, Department of Periodontics, MGM Dental College and Hospital, Navi Mumbai, Maharashtra, India

\section{Himani Gupta}

Senior Lecturer, Department of Periodontics, MGM Dental College and Hospital, Navi Mumbai, Maharashtra, India 